From an inspection of these curves, it is seen that beyond $3 \mu$, these glasses absorb most of the incident radiation. This absorption increases rapidly as the wavelength is increased beyond $3 \mu$, so that in the neighborhood of $4 \mu$, the absorption is becoming nearly complete. Near the visible end of the spectrum, many of these glasses show selective absorption to a more or less pronounced degree. In the region of the spectrum, lying very roughly between I.75 $\mu$ and $2.75 \mu$, there is approximately uniform transmission for most of the glasses studied. The absorption in the Pfund glass in the infra-red for all wavelengths was so large that it was impossible to detect with our apparatus the energy transmitted. So far as the infra-red region of the spectrum is concerned, it absorbs much more than any of the other glasses.

Physical Laboratory

Ohio State University

\title{
THE DESIGN OF LENSES FOR AERIAL PHOTOGRAPHY
}

By C. W. FredERICK

It is not the object of this paper to suggest anything new or good in lens design, nor to propose desirable modifications to fulfil the requirements of aerial photography, but merely to describe the lenses we have actually produced in the face of wartime difficulties. When the European war broke out, the supply of glass from France and England was cut off, and American manufacturers succeeded only in producing those glasses suitable for visual instruments, such as binoculars and gunsights, but failed completely to produce the dense barium crown required in high-grade photographic objectives. Thus we were confronted with the problem of making a lens of these very unsuitable materials, a problem not unlike that of making something out of nothing, and altogether discouraging to contemplate.

But first, and, as it proved, even more difficult was the necessity of finding out what was wanted in aerial photography. There was no use in guessing, for even a good guess was certain not to coincide with the ideas of others; the only thing was to find out what was actually being done. But until the United States entered the war and for a time afterward the developments in aerial photography were carefully hidden behind a military veil through which at least some of us in America could not see; and for a long time all our efforts were futile to get at the simple but necessary details as to 
lens speed, focal length, and the size of the picture to be covered. First we heard the French were using a lens of speed $f: 6.0$, but nothing as to focal length or picture. The only two glasses then availab!e at home, actually being manufactured, not merely promised, were baryta flint and dense flint. With these we tried to make a $f: 6.0$ lens. But though we succeeded in working out several good anastigmat lenses of less speed, we failed to get a $f: 6.0$ lens.

About this time we obtained the first definite information as to what the British army was doing. They were using a lens of relative aperture $f: 4.5$ with color screens, focal length 8.5 inches, and covering a plate 4 inches by 5 inches. Also from another source we were given to understand that barium glass might soon be had from abroad. Thus for the first time, we had a solid foundation with which to make a start. Selecting not the most suitable glass, but that most likely to be produced, we began work on our first f:4.5 lens, which was of the four-piece, air-spaced type.

Since these lenses were to be used with color screens which would cut out the violet light, the color curve of the lens was modified to favor the visual portion of the spectrum allowing the violet light to stray. The actual adjustment was such as to make the focal length of the lens for the yellow sodium or D line of the spectrum the same as that for the hydrogen blue or $\mathrm{G}^{\prime}$ line of the spectrum.

Another departure from the usual type of photographic lens was suggested by the relatively small area of the plate to be covered. In most lenses of the anastigmat type there is a region well out from the center of the picture where the lens gives images free from astigmatism, and where, if the lens has no coma, the image of a point source of light is as round and perfect as that formed in the center of the picture. Usually this region is from $23^{\circ}$ to $26^{\circ}$ from the optical axis of the lens, and in the case of aeroplane cameras, it is well beyond the limits of the plate. Therefore a change was made to throw the point of no astigmatism nearer to the optical axis and within the area of the picture. Other advantages were also gained by this change; it made possible a better correction for spherical aberration, and led to a form which was less sensitive to mechanical imperfection in manufacture. The latter is an important advantage in the hurry of war work.

The adjustment for spherical aberration, and even color, is not of very great importance as affecting definition. The image of a point source of light in the optical axis of a reasonably well-made lens is always too good, 
and it is a mere waste of time to split hairs over this correction. It is practically impossible to focus a lens correctly, especially in the case of aeroplane cameras which are subject to changes of temperature and rough handling, so we never really get the benefit of the central definition that exists in the lens, and it is not likely that the aberration could be so bad as to affect greatly the out of focus image. The actual adjustment for spherical aberration was such that, for a lens of $100 \mathrm{~mm}$ focal length, the extreme ray or that traversing the lens at $f: 4.5$ would strike the optical axis at a point $0.4 \mathrm{~mm}$ beyond the focal plane, and a ray traversing the lens at $f: 6.3$ would strike that axis $0.4 \mathrm{~mm}$ short of the focal plane. However, the actual image formed was always much smaller than this adjustment would indicate.

Coma, like spherical aberration, need only be reasonably well eliminated; the presence of a little coma is sometimes beneficial rather than injurious. However, since it was easy to do in a four-piece air-spaced lens, the sine condition was satisfied within $0.03 \mathrm{~mm}$ for all rays.

Distortion is important. When the lens is used for mapping it is necessary that all parts of the picture should maintain their proper relations to one another in order that their corresponding points may be accurately located on the ground. In our lens, this correction was reduced to less than o.o I $\mathrm{mm}$ at the extreme corner of the plate.

But the most difficult, and the most important of all the corrections of a lens is astigmatism. The quality of the lens depends upon the magnitude of its astigmatism. If we image the diagram of a lens in longitudinal section, with oblique rays of light traced through it, we should expect them to come to a focus at some point dependent on the components of curvature encountered in their passage through the elements of the lens. This is called the primary focus. But if we think of the rays in a plane perpendicular to the plane of the diagram, we may easily expect them to encounter different components of curvature and therefore to be brought to a different focus. This is called the secondary focus. Thus the rays of light emanating from a distant point source will not be brought to a point image. The best image or nearest approach to a point will lie somewhere between these two focal points, and it will usually be quadrilateral in form. The nearer the focal points are to each other, the smaller the best image will be. And when we speak of astigmatism, we are usually thinking of the distance from one focal point to the other, and thus indirectly of the size of the best image which controls the definition of the lens. The great problem in lens design- 
ing is to reduce the astigmatism to the lowest possible limits and to make the best images for all oblique rays fall upon the focal plane. In our first lens, we managed to keep the best images upon the focal plane within twotenths of one per cent of the focal length, and the maximum astigmatism was seven-tenths of one percent of the focal length. The properties of this lens are shown in the diagram (Fig. I). These are actual measurements on a manufactured lens, not the theoretical properties. It was finally manufactured as a ro-inch lens instead of 8.5 inches focal length as originally intended.

Before we had completed the computations on the $f: 4.5$ lens a call came for the $f: 6.0$ lens; it was wanted with a focal length of 20 inches, and wanted right away. It happened that one of the Kodak lenses we were manufacturing in smaller sizes had all the requirements for an aviation lens, except it covered a larger picture than necessary, and gave a slightly inward curving field when the astigmtism was pinched to the required amount. But since we did not have time to recompute it, the formula was multiplied up to give a focal length of 20 inches, and used without other changes than those which could be effected by varying the air spaces as required in adjusting the individual lenses in the course of manufacture. The properties of this lens as measured from an actual manufactured objective are shown in Fig. 2.

This lens was afterward recomputed to work at $f: 5.6$ and its astigmatism reduced to less than seven-tenths of one per cent. of the focal length. But the war ended before a first sample could be made.

After finishing the ro-inch $f: 4.5$ lens, though we still kept to the fourpiece air-spaced type of lens, radical changes were made in the form of the components, the resu.t being a lens in which the maximum astigmatism was less than five-tenths of one per cent. The first sample of this lens was not finished until after the armistice had been signed, and the lens was never manufactured, although it was remarkably fine. Its properties are shown in Fig. 3.

Another $f: 4.5$ formula was computed with light. barium crown and light flint, two glasses which were being manufactured in America, but through a misunderstanding as to the properties of the glass, the formula had to be recomputed, and the change led to awkward forms that were hard to get over, and the formula was only finished as the war ended. It was not very good, as the maximum astigmatism was about eight-tenths of one per cent., too much for a first-class $f: 4.5$ lens. 
Another very interesting lens was one of 48 inches focal length. A speed of only $f: 8.8$ could be obtained with the largest discs of glass that could be obtained. The formula was a modification of the Kodak anastigmat $f: 7.7$ formula, in which the components were made thinner to reduce the weight of glass in so large a lens; also the length over all was increased to flatten the aberration curve so that the same formula could be used at different apertures, as the latter would vary with the size of glass discs that could be obtained. The spherical aberration was so good that the lens would have worked at any aperture up to $f: 7.0$. Also the field was so small, only seven and a half degrees, so that astigmatism was not a problem.

The lenses that were actually manufactured in quantity were the Io-in. $f: 4.5$ lenses, and the 20-in. f:6.o. When these were mounted according to the formula they were found to vary in focal length more than allowable, and the spherical aberration and astigmatism were variable and never quite right. But here is the great advantage of a four-piece airspaced type of lens. The focal length, spherical aberration, astigmatism, and distortion can all be adjusted independently by changing the air-spacing of the components. All the aviation lenses manufactured by us were individually measured and adjusted upon the lens bench. The spherical aberration was adjusted by observing the central image, and this could always be made better than theory. Astigmatism was adjusted to the theoretical amount demanded by the formula. Distortion was never much out, and I believe it was never necessary to correct it.

Although we only manufactured two types of lenses we had formulae ready for four others, and they would have immediately gone into work if the end of the war had not brought things to a sudden stop. However, the labor directly expended to bring these six forms to final completion was only a small fraction of the total work done in the search for them. Many promising leads proved absolute failures, and many very good theoretical lenses had to be discarded because they were mechanically impossible or sometimes because they might have been awkward or troublesome to manufacture. In lens making as in everything else it is hard to make theory and practice agree, and at least three-fourths of our effort was expended to reconcile these two antagonistic and dictatorial taskmasters.

Research Laboratory

EASTMAN Kodak CoMpany

Rochester, N. Y.

December, 1918 